B. Bicsák, I. Grigoriev, S. Pop

Kliniken Maria Hilf GmbH, Akademisches Lehrkrankenhaus der Uniklinik RWTH Aachen, Mönchengladbach, Deutschland

\title{
Fieber, Kopfschmerz und fokale Anfälle bei einem 42-jährigen Mann
}
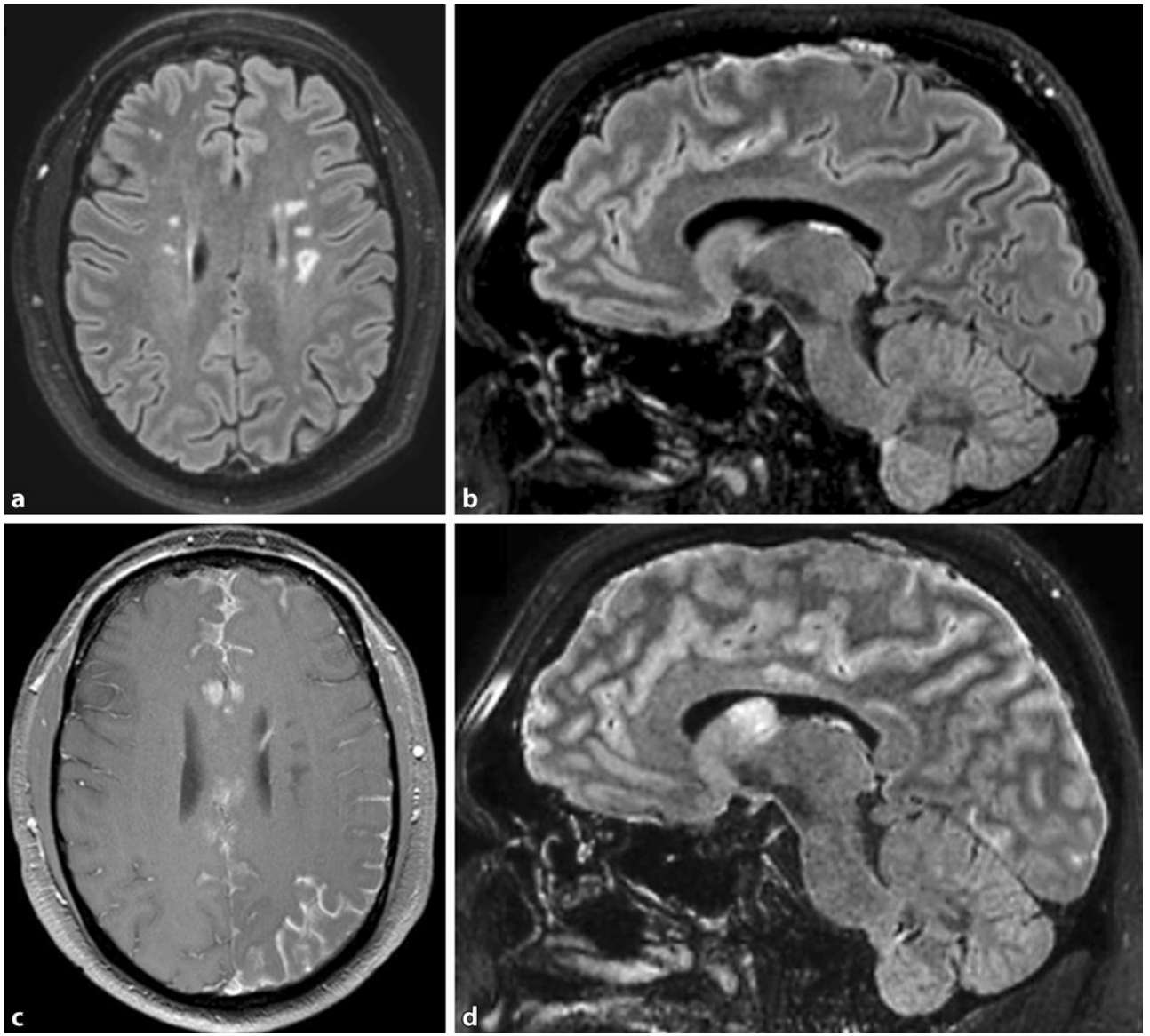

Abb. 1 a cMRT axial, FLAIR, b cMRT sagittal, FLAIR, c CMRT axial, T1+KM, d cMRT sagittal, FLAIR

\section{Auflösung des Neuro-Quiz aus DGNeurologie 1/2021}

Dieser Beitrag beinhaltet die Auflösung des Neuro-Quiz aus DGNeurologie 1/2021: Bicsák B et al. (2021) Fieber, Kopfschmerz und fokale Anfälle bei einem 42-jährigen Mann. DGNeurologie 4: https://doi.org/10.1007/s42451020-00284-5.

\section{Redaktion}

J. Becker, Essen

F. Hoffmann, Halle (Saale)

DGNeurologie $2021 \cdot 4$ (2): 117-120

https://doi.org/10.1007/s42451-020-00285-4

Online publiziert: 18. Dezember 2020

(c) Springer Medizin Verlag GmbH, ein Teil von Springer Nature 2020
Ein 42-jähriger Mann stellte sich im frühen März in unserer Notaufnahme vor, nachdem er fremdanamnestischen Angaben zufolge einen Spracharrest mit starrem Blick sowie tonischer Verkrampfung des rechten Arms erlitten hatte. Bei der Untersuchung war er klinisch internistisch und neurologisch unauffällig, dabei leicht subfebril $\left(37,5^{\circ} \mathrm{C}\right)$. An Vorerkrankungen waren eine arterielle Hypertonie und ein Diabetes mellitus bekannt; die Anamnese war hinsichtlich eines Infektgeschehens oder rezenter Impfung negativ. Im Labor fiel ein erhöhter CRP-Wert von 3,5mg/dl auf. Die kraniale Magnetresonanztomographie (cMRT) erbrachte dann ein Läsionsmuster mit periventrikulären „fingerförmigen“ T2- und FLAIR-Signalhyperintensitäten (FLAIR: „fluid attenuated inversion recovery“) im periventrikulären Marklager unter Aussparung des Balkens (Abb. 1a). Es bot sich zusätzlich eine kortikale Hyperintensität linkshemisphärisch (Abb. 1b). Der Liquorbefund zeigte eine leichte Pleozytose (8 Zellen/ $\mu$ l) und war negativ hinsichtlich Reiber-Schema, oligoklonaler Banden und erregerspezifi- 
scher Antikörperindizes. Das EEG registrierte keine epilepsietypischen Potenziale. Nach Einleitung einer Therapie mit Levetiracetam wurde er nach 6 Tagen mit der Verdachtsdiagnose einer symptomatischen Epilepsie bei erstdiagnostizierter chronisch-entzündlicher ZNS-Erkrankung aus unserer Klinik entlassen. Nach unauffälligem Verlauf über 1 Woche wurde der Patient erneut vorgestellt, diesmal mit einem passageren Taubheitsgefühl und Schwäche im rechten Bein für 15 min. Zusätzlich klagte er über linksseitige Kopfschmerzen und hatte febrile Temperaturen $\left(38,2^{\circ} \mathrm{C}\right)$. Klinisch-neurologisch war der Patient bei der Aufnahme unauffällig. Im Labor war das CRP erhöht $(10,8 \mathrm{mg} / \mathrm{dl})$. Das cMRT zeigte diesmal eine meningeale Kontrastmittelanreicherung (Abb. 1c) und anstelle der zuvor gesichteten fokalen Läsion ein ausgedehntes, kortikales Ödem der linken Hemisphäre (Abb. 1d). Die erneute Liquordiagnostik erbrachte eine Pleozytose $(249 / \mu \mathrm{l})$, erhöhte Werte für Gesamteiweiß $(107,2 \mathrm{mg} / \mathrm{dl})$ bei grenzwertigem Laktat $(2,5 \mathrm{mmol} / \mathrm{l})$ und normwertiger Glukose $(62,0 \mathrm{mg} / \mathrm{dl})$.

\section{Wie lautet lhre Diagnose?}

\section{I) Diagnose: Akute disseminierte Enzepha- lomyelitis (ADEM) - im Rahmen einer MOG- Antikörper-assoziierten Erkrankung („MOG- antibody disease" [MOG-AD])}

\section{Differenzialdiagnose, Therapie und Diskussion}

Syndromatisch war das klinische Bild einer nichteitrigen Meningoenzephalitis zuzuordnen. Differenzialdiagnostisch kam auch ein maligner Prozess in Frage. Ein Infektfokus außerhalb des zentralen Nervensystems (ZNS) war nicht nachweisbar. Kurz zurückliegende Atemwegs- oder gastrointestinale Erkrankungen wurden nicht berichtet. Nach der stationären Aufnahme entwickelte der Patient eine anhaltende Parese und Hypästhesie im rechten Bein sowie kognitive Störungen. Therapeutisch begannen wir mit einer breiten antibiotisch-antiviralen Therapie mit Ceftriaxon, Ampicillin und Aciclovir. Nach ausbleibender Besserung wiederholten wir die Liquordiagnos-

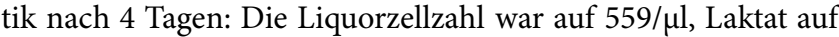
$3,0 \mathrm{mmol} / \mathrm{l}$ angestiegen bei weiterhin bestehender Proteinerhöhung (Gesamteiweiß 97,5 mg/dl). Die cMRT-Kontrolle nach 7 Tagen zeigte eine ausgedehnte linkshemisphärische kortikalleptomeningeale Entzündungsreaktion mit lokalem Hirnödem. Unverändert blieben die periventrikulären Entmarkungsherde. Im zervikalen und thorakalen Myelon waren keine Läsionen nachweisbar. Bis zum Erhalt der weiteren Ergebnisse wurde die Therapie um Amphotericin B und hochdosiertes Prednisolon (500 mg/Tag) erweitert.

Erregerspezifische Antikörperindizes (bezüglich Borrelien, VZV, HSV, HIV, Masern und Röteln) erwiesen sich erneut negativ, die quantitative TPHA war in Liquor und Serum negativ. Negativ fielen die PCR-Proben für SARS-CoV-2, Influenzavirus, RSV, Enteroviren, Toxoplasma gondii und der QuantiferonTest für mykobakterienspezifische T-Lymphozyten aus. Oligoklonale Banden waren diesmal im Liquor positiv, Antikörper gegen Aquaporin 4 waren nicht nachweisbar. Wiederholte liquorzytologische Untersuchungen erbrachten eine gemischtzel- lige Entzündungsreaktion ohne Tumorzellen oder Pilzhyphen. Wiederholte Blutkulturen zeigten kein Wachstum von Bakterien oder Pilzen. Paraneoplastische und antineurale Antikörper waren negativ, auch eine Tumorsuche mittels CT-Untersuchung des Thorax und Abdomens blieb ohne Malignitätshinweis. Zum Ausschluss einer Endokarditis wurde eine transösophageale Echokardiographie durchgeführt, mit unauffälligem Befund. Die erweiterte Diagnostik unter der Annahme einer autoimmun demyelinisierenden Genese konnte einen deutlich erhöhten Titer der MOG-AK (IgG-Antikörper gegen Myelinoligodendrozytenglykoprotein mittels ,live cell assay“) im Liquor nachweisen (1:4, Referenz: negativ), bei einem nur schwach positiven Titer (1:40, Referenzbereich: <1:20.) im Serum. Die Kontrolluntersuchung zeigte ebenfalls diese ungewöhnliche Konstellation mit positivem Liquor (1:4) bei grenzwertig positivem (1:20) Serumbefund.

Bei konstant negativer Erregerdiagnostik wurde die antibakterielle, antivirale und antimykotische Medikation abgesetzt und zunächst, ausgehend von einer akuten demyelinisierenden Enzephalomyelitis (ADEM), Prednisolon hochdosiert (500 mg/Tag) weitergeführt. Bei Stabilisierung und einer langsamen, jedoch deutlichen Besserung wurde auf die Eskalation auf i.v. Immunglobuline (IVIG) oder Plasmapherese verzichtet [1]. Im Verlauf gaben wir Prednisolon in absteigender Dosierung weiter. Korrelierend mit dem klinischen Befund war MR-tomographisch die kortikale, leptomeningeale Entzündung deutlich rückläufig, wobei die periventrikulären Marklagerläsionen unverändert blieben. Auch die Pleozytose im Liquor bildete sich nach 3 Wochen zurück $(66 / \mu \mathrm{l})$. Es gelang mit physiotherapeutischer Unterstützung die Mobilisierung am Rollator, eine Anschlussrehabilitation war aufgrund persistierender Defizite erforderlich.

Unsere Empfehlung für die nachbehandelnden Kollegen war die Fortsetzung der Steroidtherapie sowie der anfallsprophylaktischen Behandlung bis zur geplanten Kontrolle in 6 Monaten in unserer Klinik [2]. Für das weitere Vorgehen werden neben dem klinischen Verlauf und individuellen Komorbiditäten die Bildgebung und der MOG-AK-Status ausschlaggebend sein.

Für die Diagnose einer ADEM, insbesondere bei Erwachsenen, stehen bislang keine allgemein anerkannten Kriterien fest. Die angenommene Assoziation und insbesondere die Kausalität mit einem vorangegangenen Infekt oder Impfung lassen sich in den meisten Fällen schwer nachweisen. Fieber und Kopfschmerzen werden bei Kindern, sensomotorische Defizite bei Erwachsenen mit ADEM jeweils häufiger berichtet [1]. Eine der wichtigsten Differenzialdiagnosen ist die Erstmanifestation einer multiplen Sklerose (MS), hier bringt oft erst der Verlauf eine Klärung [3]. Unser Fall war aufgrund des atypischen Läsionsmusters (Aussparung des Balkens, meningeale Beteiligung) und ausgeprägter Pleozytose gut von einer MS abzugrenzen. Demyelinisierende Marklagerläsionen unterschiedlichen Alters waren in unserem Fall gleichzeitig vorhanden, diese wurden - wie auch die oligoklonalen Banden - bei einer ADEM bereits häufiger beschrieben [4]. Die Erstmanifestation einer chronisch-entzündlichen ZNS-Erkrankung im Sinne einer MOG-Enzephalomyelitis bleibt dabei als Differenzialdiagnose bestehen. Die diagnostische Entscheidung ist insbesondere im Hinblick auf das Rezidivrisiko und die Langzeitprophylaxe von Bedeutung. Entgegen der früheren Auffas- 


\begin{tabular}{l} 
Tab. 1 Diagnostische Kriterien für MOG-Enzephalomyelitis nach \\
López-Chiriboga et al. [8] \\
\begin{tabular}{l|l} 
1. & $\begin{array}{l}\text { Positive zellbasierte Detektion spezifischer Antikörper gegen MOG } \\
\text { im Serum oder Liquor }\end{array}$ \\
\hline 2. & Eines der nachfolgenden klinischen Syndrome \\
a. ADEM \\
b. Optische Neuritis (auch CRION, chronisch-rezidivierende \\
inflammatorische optische Neuropathie)
\end{tabular} \\
$\begin{array}{l}\text { c. Querschnittsmyelitis } \\
\text { d. Zerebrale demyelinisierende Erkrankung }\end{array}$ \\
$\begin{array}{l}\text { e. Kombination der oben genannten } \\
\text { 3. Ausschluss alternative Diagnosen }\end{array}$ \\
\hline Alle 3 Kriterien müssen erfüllt sein
\end{tabular}

sung, wonach eine MOG-Enzephalomyelitis als Subgruppe der MS oder der AQP-4-negativen NMO-Spektrum-Erkrankungen (NMOSD) galt, hat sie sich in den letzten Jahren als neue Krankheitsentität etabliert, die ein breites Spektrum entzündlicher ZNS-Erkrankungen umfasst [5, 6]. Eine ADEM-ähnliche Verlaufsform kommt bei Erwachsenen eher selten vor [7]. Da für die Diagnosestellung von MOG-Enzephalitiden noch keine etablierten Kriterien existieren, folgten die Autoren dem Vorschlag von López-Chiriboga et al. [8], die beim dargestellten Fall erfüllt sind (Tab. 1). Zur MOG-AK-Testung wurden die Empfehlungen des internationalen Expertenkonsenses aus 2018 [9, 10] berücksichtigt. Die ungewöhnliche MOG-Antikörper-Konstellation im Serum vs. Liquor sowie die MS-typischen, periventrikulären Marklagerläsionen sollten uns im Sinne von „red flags“ zur Vorsicht gemahnen [9, 10]. Die MOG-AK werden in der Peripherie gebildet, somit wäre eine höhere Konzentration im Serum zu erwarten. Die pathogenetische Bedeutung der MOG-AK liegt in der Immunreaktion gegen die Myelinscheide, es folgen eine Störung der Blut-Hirn-Schranke und Zerstörung von Oligodendrozyten durch die pathologische Immunantwort.

Zusammenfassend gehen wir von einer ADEM aus, im Rahmen einer MOG-Antikörper-assoziierten Erkrankung. Hierbei handelt es sich um ein neues Spektrum der Autoimmunkrankheiten des ZNS, deren nosologische Einteilung noch nicht abgeschlossen ist.

Bei einer ADEM ist der Verlauf meist monophasisch, in unserem Fall lässt die kurze Beobachtungszeit noch keine Aussage zu. Der klinische Verlauf und die Kontrolle der MOGAK im Serum (Expertenmeinungen zufolge nach 6 Monaten [2]) sollten diesbezüglich Klärung bringen. Eine negative Serokonversion begünstigt den monophasischen Verlauf, wobei bei persistierender Seropositivität der MOG-AK von einem hohen Rezidivrisiko auszugehen ist $[2,6,11]$.

Bei aktiver Krankheit gilt die Steroidpulstherapie als etabliert, bei Therapieversagen sind eine Eskalation auf i.v. Immunglobuline (IVIG) oder Plasmapherese und ggf. eine additive Gabe von Immunsuppressiva möglich $[1,2]$. $\mathrm{Zu}$ beachten ist das erhöhte Risiko bei frühzeitigem ( $<3$ Monate) Absetzen der Steroidtherapie [2, 11-14], dies gilt es zu vermeiden. Eine prophylaktische Therapie ist mit niedrigdosiertem Prednisolon, ggf. überlappend mit Immunsuppressiva wie Azathioprin, Methotrexat, Rituximab oder Mykophenolatmofetil empfohlen. Weitere Therapiemöglichkeiten bieten i.v. Immunglobu- line, Glatiramerazetat, Betainterferon und Natalizumab - alle diese Ansätze unter Beachtung von deren Nebenwirkungsprofil und der schwachen Evidenzlage [2, 11-14].

Die persistierende MOG-AK-Seropositivität wurde in einer Studie [15] auch als Risikofaktor für Anfallsrezidiv bei Kindern mit Post-ADEM-Epilepsie beschrieben. Inwieweit diese Ergebnisse auf Erwachsene extrapoliert werden können, ist bislang nicht untersucht.

\section{Fazit für die Praxis}

- Der Fall demonstriert die schwierige Differenzialdiagnose und in manchen Fällen den dramatischen Verlauf einer ADEM bei Erwachsenen.

- Unter den möglichen Ansätzen ist die Steroidpulstherapie mit langsamer Dosisreduktion am besten durch Studien belegt.

Literatur

\section{Verwendete Literatur}

1. Stüve O, Nessler S, Hartung HP et al (2005) Akute disseminierte Enzephalomyelitis Pathogenese, Diagnose, Behandlung und Prognose. Nervenarzt 76(6):701-707

2. Wynford-Thomas R, Jacob A, Tomassini V (2019) Neurological update: MOG antibody disease. J Neurol 266(5):1280-1286

3. Thompson AJ, Banwell BL, Barkhof F et al (2018) Diagnosis of multiple sclerosis: 2017 revisions of the McDonald criteria. Lancet Neurol 17(2):162-173

4. Schwarz S, Knauth M, Mohr A et al (2001) Akute disseminierte Enzephalomyelitis (ADEM). Nervenarzt 72(4):241-254

5. Di Pauli F, Berger T (2018) Myelin oligodendrocyte glycoprotein antibody-associated disorders: toward a new spectrum of inflammatory demyelinating CNS disorders? Front Immunol 9:2753

6. Cobo-Calvo A, RuizA, Maillart Eet al (2018) Clinical spectrum and prognostic value of CNS MOG autoimmunity in adults: the MOGADOR study. Neurology 90(21):e1858-e1869

7. Lana-Peixoto MA, Talim N (2019) Neuromyelitis Optica spectrum disorder and antiMOG syndromes. Biomedicines 7(2):42

8. López-Chiriboga AS, Majed M, Fryer J et al (2018) Association of MOG-lgG serostatus with relapse after acute disseminated encephalomyelitis and proposed diagnostic criteria for MOG-IgG-associated disorders. JAMA Neurol 75(11):1355-1363

9. Jarius S, Paul F, Aktas O et al (2018) MOG encephalomyelitis: international recommendations on diagnosis and antibody testing. J Neuroinflammation 15(1):134

10. Jarius S, Paul F, Aktas O et al (2018) MOG-Enzephalomyelitis: Internationale Empfehlungen zu Diagnose und Antikörpertestung. Nervenarzt 89(12):1388-1399

11. Jurynczyk M, Messina S, Woodhall MR et al (2017) Clinical presentation and prognosis in MOG-antibody disease: a UK study. Brain 140(12):3128-3138

12. Jarius S, Ruprecht K, Kleiter l et al (2016) MOG-lgG in NMO and related disorders: a multicenter study of 50 patients. Part 2: Epidemiology, clinical presentation, radiological and laboratory features, treatment responses, and long-term outcome. J Neuroinflammation 13, 280 (2016). https://doi.org/10.1186/s12974-016-0718-0

13. Borisow N, Mori M, Kuwabara Set al (2018) Diagnosis and treatment of NMO spectrum disorder and MOG-encephalomyelitis. Front Neurol 9:888

14. Dos Passos GR, Oliveira LM, da Costa BK et al (2018) MOG-IgG-associated optic neuritis, encephalitis, and myelitis: lessons learned from neuromyelitis Optica spectrum disorder. Front Neurol 9:217

15. Rossor T, Benetou C, Wright S et al (2020) Early predictors of epilepsy and subsequent relapse in children with acute disseminated encephalomyelitis. Mult scler 26(3):333-342

Weiterführende Literatur

16. Waldman AT, González-Scarano F (Ed), Dashe JF (Ed) (2020) Acute disseminated encephalomyelitis (ADEM) in adults. UpToDate 


\section{Neuro-Quiz}

\section{Korrespondenzadresse}

Dr. B. Bicsák

Kliniken Maria Hilf GmbH, Akademisches

Lehrkrankenhaus der Uniklinik RWTH Aachen

Viersener Straße 450, 41063 Mönchengladbach,

Deutschland

bertalan.bicsak@mariahilf.de

\section{Einhaltung ethischer Richtlinien}

Interessenkonflikt. B. Bicsák, I. Grigoriev und S. Pop geben an, dass kein Interessenkonflikt besteht.

Für diesen Beitrag wurden von den Autoren keine Studien an Menschen oder Tieren durchgeführt. Für die aufgeführten Studien gelten die jeweils dort angegebenen ethischen Richtlinien.

Hier steht eine Anzeige.

\section{Springer}

